TITLE:

\title{
Algebraically independent generators of invariant differential operators on a symmetric cone
}

$\operatorname{AUTHOR}(S):$

野村, 隆昭

CITATION:

野村, 隆昭. Algebraically independent generators of invariant differential operators on a symmetric cone. 数理解析研究所講究録 1990, 712: 66-72

ISSUE DATE:

1990-02

URL:

http://hdl.handle.net/2433/101697

RIGHT: 


\title{
Algebraically independent generators of invariant differential operators on a symmetric cone
}

\author{
京大理 野 柖 (Takaaki NomURA)
}

よく知られを例から始めよう． [8], [12] 参照. 実 $r \times r$ 対称行列のなすべクトル空間を $\operatorname{Sym}(r, \mathbf{R})$ で表す. $\operatorname{Sym}(r, \mathbf{R})$ は自然な内積 $\langle x, y\rangle:=\operatorname{tr}(x y)$ を持つ. 正定値なもの全体から 成る $\operatorname{Sym}(r, \mathbf{R})$ の部分集合を $\Omega$ とする. $\Omega$ は $\operatorname{Sym}(r, \mathbf{R})$ の開凸錐で，しかも内積 $(\cdot, \cdot\rangle$ K関 して自己双対である.すなわち，

$$
\Omega=\{y \in \operatorname{Sym}(r, \mathbf{R}) ;\langle x, y\rangle>0 \text { for } \forall x \in \bar{\Omega} \backslash\{0\}\}
$$

$\left(\bar{\Omega}\right.$ は $\Omega$ の閉包) が成り立つ. 線型 Lie 群 $G L(r, \mathbf{R})$ は $\operatorname{Sym}(r, \mathbf{R})$ K $x \mapsto g x^{t} g$ とより作用 していて, $\Omega$ は乙の作用による単位行列 $e \in \Omega$ の $G L(r, \mathbf{R})$-軌道Kなっている. $\Omega$ 上の微分作 用素で, $G L(r, \mathbf{R})$ の作用と可換をものの全体を $\mathbf{D}(\Omega)^{G L(r, \mathbf{R})}$ とする. とのとを， $r$ 個の微分作 用素

$$
\operatorname{tr}\left(\left(x \frac{\partial}{\partial x}\right)^{j}\right) \quad(j=1,2, \ldots, r)
$$

は $\mathbf{D}(\Omega)^{G L(r, \mathbf{R})}$ の代数的飞独立な生成元になっている.

本稿では，ての結果を任意の自己双対な開凸錐(以下対称錐と呼ぶ) K，その分類を用らずしか も explicit な形で,一般化する. そのために, 1950 年代の終わりК, Koecher [7] や Vinberg [13] とよって発見された事実一任意の対称錐はある種のJordan 代数によって記述される一 を用斿。

\section{§1. Jordan 代数}

Jordan 代数の定義から始め上5. 証明は [1], [3], [5], [6], [11] 等を見られたい. 実べク トル空間 $V$ K次の (1),(2) をみをす双線型写像(すなわち, 積) $V \times V \ni(x, y) \mapsto x y \in V$ が 定義されているとき, $V$ を赛 Jordan 代数 とい5: $\forall x, y \in V$ K対して,

(1) $x y=y x$,

(2) $x^{2}(x y)=x\left(x^{2} y\right)$.

とてでは, 結合律は仮定されていならととに注意. 各 $x \in V$ 凡対して, 作用素 $L(x)$ を

$$
L(x) y=x y \quad(y \in V)
$$


で定義する. 2 つの作用素 $A, B$ K対して, $[A, B]=A B-B A$ とおくと,(2) は作用素の等式 として

$$
\left[L(x), L\left(x^{2}\right)\right]=0
$$

と書き直せるととに注意しておく。

さて, Jordan 代数 $V$ は非結合的代数であるが, べを関しては指数法則が成り立っている (との意味で, Jordan 代数は power associative algebra Kなっている). そして, $V$ 上の $i$ 次の斉次多項式函数 $\sigma_{i}(1 \leqq i \leqq d)$ が存在して

$$
m_{x}(\lambda)=\lambda^{d}-\sigma_{1}(x) \lambda^{d-1}+\sigma_{2}(x) \lambda^{d-2}+\cdots+(-1)^{d} \sigma_{d}(x)
$$

が, $V$ のある Zariski-dense な開集合に属する $\forall x$ 凡対して, $x$ の最小多項式になるとと,及び $m_{x}(\lambda)$ の既約成分は, $x$ の最小多項式の 1 つの因数になっているてとが知られている [5]. 多項 式 $m_{x}(\lambda)$ は $x \in V$ の一般最小多項式と呼ばれ, $d$ を $V$ degree という. 以下では,

$$
T(x)=\sigma_{1}(x)
$$

とおいて, 線型形式 $T$ を $V$ の trace と呼ら゙とととする.

実 Jordan 代数 $V$ Kおいて,

$$
x^{2}+y^{2}=0 \quad \text { ならば } \quad x=y=0
$$

が成り立つとき，Vは 形式的実であるという. 形式的実 Jordan 代数は必ず単位元 $e$ を持つ. 以下, Jordan 代数 $V$ は形式的実であるとする. $V$ の 0 でないべを等元の系 $e_{1}, e_{2}, \ldots, e_{k}$ が 直交系であるとは, $i \neq j$ ならば $e_{i} e_{j}=0$ が成り立つととであり，それが完全であるとは， $e_{1}+\cdots+e_{k}=e($ 単位元 $)$ となるととである.

命題1.1(スベクトル分解)ＶVの各元 $x$ 凡対して, 0 でないべき等元の完全直交系 $e_{1}, e_{2}, \ldots, e_{k}$ と実数 $\lambda_{1}, \ldots, \lambda_{k}\left(\lambda_{1}<\lambda_{2}<\cdots<\lambda_{k}\right)$ が存在して, $x=\lambda_{1} e_{1}+\cdots+\lambda_{k} e_{k}$ と表される. それらは一意的で, $\lambda_{1}, \ldots, \lambda_{k}$ のととを, $x$ の固有值という.

0でないべき等元は原始へを等元の直交和に分解でをるから, 特に単位元 $e$ は原始べを等元の 直交和として $e=e_{1}+\cdots+e_{r}$ と，分解できるととがわかる．とてで，r は原始へき等元の完全 直交系のとり方によらず一定で， $V$ の階数と呼ばれ，上述の degree に一致する.

命題 1.2. Jordan 代数 $V$ Kおいて, 次の $(1) \sim(3)$ は同值 :

(1) $V$ は形式的実.

(2) 対称双線型形式 $x, y \mapsto \operatorname{tr} L(x y)$ は正定値.

(3) 対称双線型形式 $x, y \mapsto T(x y)$ は正定値.

例. $\operatorname{Sym}(r, \mathbf{R}) k x y=(x \cdot y+y \cdot x) / 2$ (右辺の - は通常の行列の乗法)で積を入れると， $\operatorname{Sym}(r, \mathbf{R})$ は形式的実 Jordan 代数になる. 実際, $x^{2}+y^{2}=0$ なら $x \cdot x+y \cdot y=0$ だか ら, $\forall \xi \in \mathbf{R}^{r}$ K対して

$$
0={ }^{t} \xi \cdot(x \cdot x+y \cdot y) \cdot \xi=|x \cdot \xi|^{2}+|y \cdot \xi|^{2} .
$$


とれより $x=y=0$ が出る.

$V$ の各元 $x$ 凡対して, 作用素 $P(x)$ を

$$
P(x):=2 L(x)^{2}-L\left(x^{2}\right) \quad(x \in V)
$$

で定義する. 対応 $P: x \mapsto P(x)$ は $V$ の quadratic representation と呼ばれる $(P(x y)=$ $P(x) P(y)$ は一般に成り立たないけれども). との $P$ について次の公式が成り立つ: $\forall x, y \in V$ K対して

$$
\begin{aligned}
P(P(x) y) & =P(x) P(y) P(x), \\
P\left(x^{n}\right) & =P(x)^{n} \quad(n=1,2, \ldots) .
\end{aligned}
$$

さて, $V$ の元 $x$ K対して, $x$ で生成される $V$ の部分代数を $\mathbf{R}[x]$ で表そう. $V$ は powerassociative だから, $\mathbf{R}[x]$ では結合律が成立しているととに注意.

命題 1.3. $V$ の元 $x$ K対して, 次の $(1) \sim(4)$ は同値 :

(1) 作用素 $P(x)$ は可逆, 寸なわち, $\operatorname{det} P(x) \neq 0$.

(2) $\mathbf{R}[x]$ の元 $y$ が存在して, $x y=e$.

(3) $V$ の元 $y$ が存在して; $x y=e$ かつ $[L(x), L(y)]=0$.

(4) $V$ の元 $y$ が存在して, $x y=e$ かつ $x^{2} y=x$.

命題 1.3 の条件をみたす $x$ は可逆であるといわれる. (3) より $x$ の逆元 $y$ の一意性がわか り，(1) を用いると, $y=P(x)^{-1} x$ で与えられるととがわかる. 可逆元の全体を $V^{\times}$で表す. と のとき，

$$
P\left(x^{-1}\right)=P(x)^{-1} \quad\left(\forall x \in V^{\times}\right) .
$$

$V$ Kは $\langle x, y\rangle:=T(x y)$ で内積を入れておく(命題 1.2). とのとき，任意の $x \in V$ 凡対し $\tau, L(x), P(x)$ は自己共役作用素である.

命題 1.4. 次の 5 つの $V$ の部分果合は同一である :

(1) Int $\left\{x^{2} ; x \in V\right\} \quad$ (Int は集合の内部 ).

(2) $\left\{x^{2} ; x \in V^{\times}\right\}$.

(3) $V^{\times}$の単位元の連結成分.

(4) $\{x \in V ; L(x)$ は正定値 $\}$.

(5) $\{x \in V ; x$ の固有値はすべて正 $\}$.

命題 1.4 で定義される $V$ の部分集合を $\Omega$ で表す. $\Omega$ は $V$ の開凸錐で内積 $\langle\cdot, \cdot\rangle$ 凡関して 自己双対である. $\Omega$ のととを $V$ の 対称錐 という. $x \in \Omega$ ならぼ, $P(x)$ は正定值であるととも 注意してまてう。

さて, 写像 $F: \Omega \ni x \mapsto x^{2} \in \Omega$ を考えよ5. $x_{0} \in V$ での Fréchet 微分は $2 L\left(x_{0}\right)$ で， しかもスベクトル分解を考元るととにより容易に $F$ が上への写像であるととがかかるから， $F$ は $\Omega$ から $\Omega$ の上への微分同相である. $F$ の逆写像を $\Omega \ni y \mapsto y^{1 / 2} \in \Omega$ と表す. 
$V$ 上の実一般線型群を $G L(V)$ とし

$$
G(\Omega):=\{g \in G L(V) ; g \Omega=\Omega\}
$$

とまく. $G(\Omega)$ は $G L(V)$ の閉部分群であるから, $G(\Omega)$ は Lie 群になる. $\Omega$ が自己双対であ ろので, $G(\Omega)$ は reductive な Lie 群である.

補題 1.5. $x \in V^{\times}$なら $P(x) \in G(\Omega)$.

証明. (1.1) より, $\forall y \in \Omega$ 飞対して $P(x) y$ は可逆 (i.e. $\left.\exists P(P(x) y)^{-1}\right)$ であるから $P(x) y \in$ $V^{\times}$. そして $P(x) e=x^{2} \in \Omega$ だから, $P(x) \Omega$ は $V^{\times}$の連結開集合で, $\Omega$ と共通部分を持つ. ゆえに, $P(x) \Omega \subset \Omega$. とれと (1.3) より $P(x) \Omega=\Omega$. すなわち, $P(x) \in G(\Omega)$.

任意の $x \in \Omega$ 凡対して, $P\left(x^{1 / 2}\right) e=x$ ゅえ, 補題 1.5 から特に $\Omega=G(\Omega) e$ であるとと がわかる.

\section{§2. 不変多項式}

以下, 形式的実 Jordan 代数 $V$ は単純て階数が $r$ であるものとする. $V$ の自己同型がなす 群を $K$ で表す.すなわち，

$$
K:=\{g \in G L(V) ; g(x y)=(g x)(g y) \quad \text { for } \forall x, y \in V\}
$$

一般最小多項式の $K$-不変性 : $m_{x}(\lambda)=m_{k x}(\lambda)(\forall k \in K, x \in V)$ から,$K$ は $V$ の内積 $\langle\cdot, \cdot\rangle$ 凡関する直交群 $O(V)$ の閉部分群になるととがわかる. 従って, $K$ 自身コンバクト Lie 群 である. $V$ 上の $K$-不変な多項式函数のなす代数を $\operatorname{Pol}(V)^{K}$ で表す. $\operatorname{Pol}(V)^{K}$ Kつレては, 次の結果が知られている.

命題 2.1(U. Hirzebruch [4]). $\quad r$ 個の $V$ 上の多項式函数

$$
f_{j}(x):=T\left(x^{j}\right) \quad(j=1,2, \ldots, r)
$$

は $\operatorname{Pol}(V)^{K}$ の代数的に独立な生成元である.

次の命題は J. Faraut 教授に教わった.

命題 2.2. $f \in \operatorname{Pol}(V)^{K}$ とすると, 函数

$$
\Omega \times V \ni(x, y) \mapsto f\left(P\left(x^{1 / 2}\right) y\right)
$$

は, $p(x, y)=p(y, x)(\forall x, y \in V)$ である様な $V \times V$ 上の多項式函数 $p$ の $\Omega \times V$ への制限 である.

証明は, Faraut 氏の承諾を得て, [9]凡書いておいた。 
例 1. 命題 $2.2 て ゙ f=T=f_{1}$ とすると

$$
T\left(P\left(x^{1 / 2}\right) y\right)=\left\langle P\left(x^{1 / 2}\right) y, e\right\rangle=\langle y, x\rangle
$$

であるから, $p(x, y)=\langle x, y\rangle$.

例 2.一般最小多項式の定数項の $(-1)^{r}$ 倍，すなわち， $\sigma_{r}(x)$ を $N(x)$ と書とう.もちろん, $N \in \operatorname{Pol}(V)^{K}$.とのとを，

$$
N\left(P\left(x^{1 / 2}\right) y\right)=N(x) N(y) .
$$

従って, $p(x, y)=N(x) N(y) . V=\operatorname{Sym}(r, \mathbf{R})$ のとをは, $N(x)=\operatorname{det} x, P\left(x^{1 / 2}\right) y=$ $x^{1 / 2} \cdot y \cdot x^{1 / 2}$ であるから, $(2.2)$ は $\operatorname{det}\left(x^{1 / 2} \cdot y \cdot x^{1 / 2}\right)=(\operatorname{det} x)(\operatorname{det} y)$ といら式に他なら ない.

命題 2.1 の $f_{j}$ 凡対して, $f_{j}\left(P\left(x^{1 / 2}\right) y\right)$ を例 1 の様に, explicit そ表そう.

命題 2.3. $\forall x \in \Omega$ と $\forall y \in V$ K対して,

$$
\begin{aligned}
f_{2 m-1}\left(P\left(x^{1 / 2}\right) y\right) & =\left\langle(P(x) P(y))^{m-1} x, y\right\rangle, \\
f_{2 m}\left(P\left(x^{1 / 2}\right) y\right) & =\left\langle(P(x) P(y))^{m-1} x,(y \square x) y\right\rangle .
\end{aligned}
$$

ただし, $y \square x=L(y x)+[L(y), L(x)]$.

注意. 命題 2.2 より, 命題 $2.3(1),(2)$ の右辺は, $x, y$ とつ々て対称である. 作用素 $P(x)$ が自 己共役であったから, (1) の右辺が $x, y$ 凡ついて対称なととは直接読み取れる. (2) の右辺につい ては，作用素 $y \square x$ の adjoint が $x \square y$ となるとと，及び Jordan 代数（というより Jordan 3 重系)での基本的な公式 :

$$
(x \square y) P(x)=P(x)(y \square x)
$$

からわかる.

以下，

$$
\begin{aligned}
p_{2 m-1}(x, y) & :=\left\langle(P(x) P(y))^{m-1} x, y\right\rangle, \\
p_{2 m}(x, y) & :=\left\langle(P(x) P(y))^{m-1} x,(y \square x) y\right\rangle
\end{aligned}
$$

とおく．各 $p_{j}$ は $V \times V$ 上の多項式函数であるが，さらに

命題 2.4. 各 $j=1,2, \ldots, r$ Kつレ

$$
p_{j}\left(g x,{ }^{t} g^{-1} y\right)=p_{j}(x, y) \quad \text { for } \forall g \in G(\Omega), x \in V, y \in V
$$

が成り立つ. ただし, ${ }^{t} g$ は 内積 $\langle\cdot, \cdot\rangle$ K関する $g$ の adjoint.

さて, $G(\Omega)$ を $V \times V$ K

$$
g \cdot(x, y)=\left(g x,{ }^{t} g^{-1} y\right)
$$

で作用させる。 
補題 2.5. $\Omega \times V$ 上の $C^{\infty}$-函数 $L$ は，(2.5) とよる $G(\Omega)$ の作用で不卒で，かつ各 $x \in \Omega$ を 固定するとき, $y \mapsto L(x, y)$ は $V$ 上の多項式函数であるとする. とのとき, $r$ 変数の多項式函数 $Q$ が存在して,

$$
L(x, y)=Q\left(p_{1}(x, y), \ldots, p_{r}(x, y)\right) \quad(\forall x \in \Omega, \forall y \in V)
$$

となる. ととで, $p_{j}$ は (2.3),(2.4)で定義されたものである.

証明は, $K \subset O(V)$ 飞注意して, $l(y):=L(e, y)$ で定義される $V$ 上の多項式函数 $l$ が $\operatorname{Pol}(V)^{K}$ 飞属するととと, U. Hirzebruch の結果 (命題 2.1$)$, 及び $L(x, y)=l\left(P\left(x^{1 / 2}\right) y\right)$ となるととを用いる。

$(2.5)$ Kよる $G(\Omega)$ の作用で不㚆な $V \times V$ 上の多項式函数のなす代数を $\operatorname{Pol}(V \times V)^{G(\Omega)}$ で表そう. 命題 2.2 を精密化すると

定理 2.6. 各 $f \in \operatorname{Pol}(V)^{K}$ K対して,

$$
p_{f}(x, y):=f\left(P\left(x^{1 / 2}\right) y\right) \quad(x \in \Omega, y \in V)
$$

とおくと, 写像 $f \mapsto p_{f}$ は, $\operatorname{Pol}(V)^{K}$ から $\operatorname{Pol}(V \times V)^{G(\Omega)}$ の上への algebra isomorphism を与える。

§3. $G(\Omega)$-不变微分作用素

$G(\Omega)$ の $V$ への作用は線型であったから， $G(\Omega)$ の $V \times V$ への作用 $(2.5)$ を $\Omega \times V$ 飞制限すると，それは $\Omega$ の余接束 $T^{*}(\Omega) \approx \Omega \times V$ への $G(\Omega)$ の自然な作用他ならなら. $(2.3),(2.4)$ で定義された $p_{j}$ を用的，徽分作用素 $p_{j}(x, \partial / \partial x)$ を

$$
p_{j}(x, \partial / \partial x) e^{\langle x, y\rangle}=p_{j}(x, y) e^{\langle x, y\rangle}
$$

で定義する.

定理 3.1. $r$ 個の微分作用素 $p_{1}(x, \partial / \partial x), \ldots, p_{r}(x, \partial / \partial x)$ は, $\Omega$ 上の $G(\Omega)$-不変な微分作 用素のな寸代数 $\mathrm{D}(\Omega)^{G(\Omega)}$ の代数的に独立な生成元である.

例. $x \in \Omega$ のとを, $P(x)$ は正定値自己共役作用素であったととを思䖦そ5. 各 $x \in \Omega$ K対 して

$$
B_{x}(u, v):=\langle P(x) u, v\rangle \quad(u, v \in V)
$$

とおくと, $B: x \mapsto B_{x}$ は $\Omega$ K $(\Omega)$-不変な Riemann 構造を定義し, $\Omega$ は Riemann 対称 空間となる. 点 $e \in \Omega$ での symmetry は $x \mapsto x^{-1}$ (Jordan 代数 $V$ での inverse) で与え られる. とのとを, Riemann 構造 $B$ K関する $\Omega$ 上の Laplace-Beltrami 作用素 $\Delta$ は

$$
\Delta=p_{2}(x, \partial / \partial x)+\frac{n}{r} \cdot p_{1}(x, \partial / \partial x)
$$

と表される. ととで, $p_{1}(x, y)=\langle x, y\rangle$ であったから, $p_{1}(x, \partial / \partial x)$ はレわゆる Euler 作用素 である. 


\section{REFERENCES}

1. H. Braun and M. Koecher, "Jordan-Algebren," Springer, Berlin-Heidelberg-New York, 1966.

2. J. Dorfmeister and M. Koecher, Reguläre Kegel, Jber. Deutsch. Math.-Verein. 81 (1979), 109-151.

3. J. Faraut, "Algèbres de Jordan et cônes symétriques," Ecole d'été CIMPA, Univ. Poitiers, 1988.

4. U. Hirzebruch, Invariant polynomial functions on Jordan algebras, Algebras Groups Geom. 1 (1984), 442-445.

5. N. Jacobson, "Structure and representations of Jordan algebras," Amer. Math. Soc., Providence, 1968.

6. P. Jordan, J. von Neumann and E. Wigner, On an algebraic generalization of the quantum mechanical formalism, Ann. of Math. 35 (1934), $29-64$.

7. M. Koecher, Die Geodätischen von Positivitätsbereichen, Math. Ann. 135 (1958), 192-202.

8. H. Maaß, "Siegel's modular forms and Dirichlet series," Lect. Notes in Math. 216, Springer, Berlin-Heidelberg-New York, 1971.

9. T. Nomura, Algebraically independent generators of invariant differential operators on a symmetric cone, J. Reine Angew. Math. 400 (1989), 122-133.

10. T. Nomura, Algebraically independent generators of invariant differential operators on a bounded symmetric domain, J. Math. Kyoto Univ., to appear.

11. I. Satake, ジョルダン環とリー環 I, II, III, 科学 51 (1981), 287-291; 387-391; 458-463.

12. A. Selberg, Harmonic analysis and discontinuous groups in weakly symmetric Riemannian spaces with applications to Dirichlet series, J. Indian Math. Soc. 20 (1956), 47-87.

13. E. B. Vinberg, Homogeneous cones, Soviet Math. Dokl. 1 (1961), 787-790. 